\title{
Gauge coupling unification in a classically scale invariant model
}

\author{
Naoyuki Haba, ${ }^{a}$ Hiroyuki Ishida, ${ }^{a}$ Ryo Takahashi $^{b}$ and Yuya Yamaguchi ${ }^{a, c}$ \\ ${ }^{a}$ Graduate School of Science and Engineering, Shimane University, \\ Matsue 690-8504, Japan \\ ${ }^{b}$ Graduate School of Science, Tohoku University, \\ Sendai, 980-8578 Japan \\ ${ }^{c}$ Department of Physics, Faculty of Science, Hokkaido University, \\ Sapporo 060-0810, Japan \\ E-mail: haba@riko.shimane-u.ac.jp, ishida@riko.shimane-u.ac.jp, \\ r.takahashi@m.tohoku.ac.jp, yy@particle.sci.hokudai.ac.jp
}

ABSTRACT: There are a lot of works within a class of classically scale invariant model, which is motivated by solving the gauge hierarchy problem. In this context, the Higgs mass vanishes at the UV scale due to the classically scale invariance, and is generated via the Coleman-Weinberg mechanism. Since the mass generation should occur not so far from the electroweak scale, we extend the standard model only around the TeV scale. We construct a model which can achieve the gauge coupling unification at the UV scale. In the same way, the model can realize the vacuum stability, smallness of active neutrino masses, baryon asymmetry of the universe, and dark matter relic abundance. The model predicts the existence vector-like fermions charged under $\mathrm{SU}(3)_{C}$ with masses lower than $1 \mathrm{TeV}$, and the SM singlet Majorana dark matter with mass lower than $2.6 \mathrm{TeV}$.

Keywords: Beyond Standard Model, Cosmology of Theories beyond the SM, Gauge Symmetry, Renormalization Group

ARXIV EPRINT: 1511.02107 


\section{Contents}

1 Introduction 1

$\begin{array}{llr}2 & \text { Symmetry breaking mechanism } & 2\end{array}$

3 Phenomenological and cosmological aspects 5

3.1 Gauge coupling unification 6

$\begin{array}{lll}3.2 & \text { Vacuum stability and triviality } & 7\end{array}$

$\begin{array}{ll}3.3 & \text { Neutrino masses and baryon asymmetry of the universe } \\ 3.4\end{array}$

$\begin{array}{lll}3.4 & \text { Dark matter } & 11\end{array}$

4 Conclusion 13

A $\beta$ functions in the $\mathrm{U}(1)_{X}$ extended SM 13

\section{Introduction}

The Higgs mass parameter $m_{h}^{2}$ is only a dimensionful parameter in the standard model $(\mathrm{SM})$, and its value is estimated by the observed Higgs mass as $\sqrt{-2 m_{h}^{2}}=M_{h}=125.09 \pm$ 0.21 (stat.) \pm 0.11 (syst.) GeV [1]. Then, a running of the Higgs quartic coupling becomes negative below the Planck scale within the SM. If the SM can be valid up to a high energy scale such as a breaking scale of a gauge symmetry in the grand unification theory (GUT), the electroweak (EW) scale should be stabilized against radiative corrections coming from the high energy physics. To solve the gauge hierarchy problem, there are a lot of works motivated by a classically scale invariance [2]-[29]. The scale invariance prohibits dimensionful parameters at a classical level, while it can be radiatively broken by the Coleman-Weinberg (CW) mechanism [30]. In addition to the classically scale invariance, with an additional $\mathrm{U}(1)_{X}$ gauge symmetry, e.g., $\mathrm{U}(1)_{B-L}$ gauge symmetry, it is possible to naturally realize experimentally observed values of the Higgs mass. When the $\mathrm{U}(1)_{X}$ symmetry is broken by the CW mechanism, the EW symmetry could be also broken through the scalar mixing term. If the $\mathrm{U}(1)_{X}$ breaking scale is not far from the EW scale, the Higgs mass corrections would be sufficiently small, and then the hierarchy problem can be solved. Note that these statements are based on the Bardeen's argument [31], and we consider only logarithmic divergences in this paper (see ref. [7] for more detailed discussions).

In this paper, we assume the classically scale invariance at the UV scale, where the SM gauge couplings are unified. We expect that some unknown mechanism, such as a string theory, realizes the classically scale invariance and the gauge coupling unification (GCU). Actually, the GCU can be realized at $3 \times 10^{16} \mathrm{GeV}$ in our model, and the scale is near the typical string scale $\left(\sim 10^{17} \mathrm{GeV}\right)$. To realize the GCU, some additional particles with the 
SM gauge charges are needed. Conditions of the GCU can be systematically obtained by an analysis of renormalization group equations (RGEs) [32, 33]. When all additional particles are vector-like fermions with the $\mathrm{TeV}$ scale masses, the GCU scale can be realized between $10^{16} \mathrm{GeV}$ and $10^{17} \mathrm{GeV}$, and there are a lot of possibilities to realize the GCU at the scale. ${ }^{1}$ For example, vector-like pairs of quark doublet $Q_{L, R}$ and down-type quark singlet $D_{L, R}$ can achieve the GCU $[34,35]$. When there are additional fermions charged under the SM gauge symmetries, the gauge couplings and the top Yukawa coupling respectively become larger and smaller compared to the SM case, and then, both changes make the $\beta$ function of the Higgs quartic coupling become larger. Therefore, the vacuum can become stable when the GCU is realized.

To solve the gauge hierarchy problem, there should be no intermediate scale between the EW and the GCU scales except an energy scale, which is not so far from the EW scale, i.e., the TeV scale. Then, phenomenological and cosmological problems (e.g., smallness of active neutrino masses, baryon asymmetry of the universe, and dark matter (DM)) should be explained with sufficiently small Higgs mass corrections. The first two problems can be explained by the right-handed neutrinos, which are naturally introduced to cancel the anomalies accompanied with the $\mathrm{U}(1)_{X}$ gauge symmetry, via type-I seesaw mechanism [3640] and resonant leptogenesis [41], respectively. In our model, the DM is identified with the SM singlet Majorana fermions, and its stability can be guaranteed by an additional $Z_{2}$ symmetry [42]. In this paper, we will show that our model can explain the above problems as well as realizing the GCU without affecting the hierarchy problem. ${ }^{2}$

In the next section, we will define our model, and explain the $\mathrm{U}(1)_{X}$ gauge symmetry breaking as well as the EW symmetry breaking via the $\mathrm{CW}$ mechanism. We also obtain the upper bound on the $\mathrm{U}(1)_{X}$ breaking scale from the naturalness. In section 3, we will discuss the GCU, vacuum stability, smallness of active neutrino masses, baryon asymmetry of the universe, and the DM relic abundance. Our model predicts the existence vector-like fermions charged under $\mathrm{SU}(3)_{C}$ with masses lower than $1 \mathrm{TeV}$, and the SM singlet Majorana dark matter with mass lower than $2.6 \mathrm{TeV}$. We summarize our results in section 4 .

\section{Symmetry breaking mechanism}

We consider the $\mathrm{U}(1)_{X}$ gauge extension of the SM with three generations of the righthanded neutrinos $\nu_{R_{i}}(i=1,2,3)$, six vector-like fermions $\left(Q_{L}, Q_{R}, D_{L}, D_{R}, N_{L}\right.$, and $N_{R}$ ), and two SM singlet scalars $(\Phi$ and $S$ ). Charge assignments of the particles are shown in table 1 . The $\mathrm{U}(1)_{X}$ charge are given by $B-L+2 x_{H} Y$, where $x_{H}, B, L$, and $Y$ denote a

\footnotetext{
${ }^{1}$ For example, we can consider the origin of the vector-like fermions as the string theory, in which a number of vector-like fermions should appear above the compact scale, which is expected to be the GCU scale in our model. Some of them might have the $\mathrm{TeV}$ scale masses due to the fine-tuning of moduli (or Wilson line, extra-dimensional component of anti-symmetric tensor field, and so on).

${ }^{2}$ From theoretical point of view, there are some papers constructing a model which realizes classically scale invariance and gauge coupling unification at the same scale [43]-[45]. Furthermore, asymptotic safety of gravity [46] leads vanishing couplings at the UV scale, which suggests vanishing quartic couplings and gauge coupling unification around the Planck scale [see figure 1 in ref. [47] for example]. In this paper, we simply expect such a situation comes from unknown UV physics.
} 


\begin{tabular}{|c|c|cc|}
\hline & $\mathrm{SU}(3)_{C} \otimes \mathrm{SU}(2)_{L} \otimes \mathrm{U}(1)_{Y}$ & $\mathrm{U}(1)_{X}$ & $Z_{2}$ \\
\hline$q_{L}$ & $(3,2,1 / 6)$ & $\left(x_{H}+1\right) / 3$ & + \\
$u_{R}$ & $(3,1,2 / 3)$ & $\left(4 x_{H}+1\right) / 3$ & + \\
$d_{R}$ & $(3,1,-1 / 3)$ & $\left(-2 x_{H}+1\right) / 3$ & + \\
$\ell_{L}$ & $(1,2,-1 / 2)$ & $-x_{H}-1$ & + \\
$e_{R}$ & $(1,1,-1)$ & $-2 x_{H}-1$ & + \\
$\nu_{R}$ & $(1,1,0)$ & -1 & + \\
$H$ & $(1,2,1 / 2)$ & $x_{H}$ & + \\
$Q_{L, R}$ & $(3,2,1 / 6)$ & $\left(x_{H}+1\right) / 3$ & - \\
$D_{L, R}$ & $(3,1,-1 / 3)$ & $\left(-2 x_{H}+1\right) / 3$ & - \\
$N_{L, R}$ & $(1,1,0)$ & -1 & - \\
$\Phi$ & $(1,1,0)$ & 2 & + \\
$S$ & $(1,1,0)$ & 0 & + \\
\hline
\end{tabular}

Table 1. Charge assignment of particles, where $x_{H}$ is a real number.

real number, the baryon and lepton numbers, and the $\mathrm{U}(1)_{Y}$ hypercharge, respectively. In particular, $x_{H}=0,-1$ and $-2 / 5$ correspond to $\mathrm{U}(1)_{B-L}, \mathrm{U}(1)_{R}$ and $\mathrm{U}(1)_{\chi}$, respectively. The vector-like fermions $Q_{L, R}, D_{L, R}$, and $N_{L, R}$ respectively have the same charges as the SM quark doublet, the SM down-quark singlet, and the right-handed neutrino, while only the vector-like fermions are odd under an additional $Z_{2}$ symmetry. Four of the vector-like fermions $\left(Q_{L, R}\right.$ and $\left.D_{L, R}\right)$ play a role for achieving the GCU, and the others $\left(N_{L, R}\right)$ are the DM candidates, whose stability is guaranteed by the $Z_{2}$ symmetry. These particles are not necessary for the realization of GCU and DM. We choose them for the simplest extension.

The relevant Lagrangian is given by

$$
\begin{aligned}
\mathcal{L}= & \mathcal{L}_{\mathrm{SM}}+\mathcal{L}_{\text {kinetic }}-V(H, \Phi, S)-\left(Y_{\nu} \overline{\ell_{L}} H^{c} \nu_{R}+\kappa_{1} \overline{Q_{L}} H D_{R}+\kappa_{2} \overline{D_{L}} H Q_{R}\right. \\
& +Y_{M} \Phi \overline{\nu_{R}^{c}} \nu_{R}+Y_{N_{L}} \Phi \overline{N_{L}} N_{L}^{c}+Y_{N_{R}} \Phi \overline{N_{R}^{c}} N_{R} \\
& \left.+f_{Q} S \overline{Q_{L}} Q_{R}+f_{D} S \overline{D_{L}} D_{R}+f_{N} S \overline{N_{L}} N_{R}+\text { h.c. }\right)
\end{aligned}
$$

where $\mathcal{L}_{\mathrm{SM}}$ is the SM Lagrangian except for the Higgs sector, $\mathcal{L}_{\text {kinetic }}$ includes kinetic terms of the Higgs and new particles, and $V(H, \Phi, S)$ is a scalar potential of the model. Without the $Z_{2}$ symmetry, there are also additional Yukawa interactions between the SM particles and the new particles, e.g., $y_{1} \overline{Q_{L}} H^{c} u_{R}, y_{2} \overline{Q_{L}} H d_{R}$, and $y_{3} \overline{q_{L}} H D_{R}$. However, these coupling constants have to be very small due to constraints from the precision electroweak data [48]. To forbid these terms, we have imposed odd parity to only the vector-like fermions under the $Z_{2}$ symmetry.

Since there are two $\mathrm{U}(1)$ gauge symmetry, $\mathrm{U}(1)$ kinetic mixing generally arises in the model. We can take covariant derivative as

$$
D_{\mu}=\partial_{\mu}+i g_{3} T^{\alpha} G_{\mu}^{\alpha}+i g_{2} T^{a} W_{\mu}^{a}+i g_{Y} Y B_{\mu}+i\left(g_{\mathrm{mix}} Y+g_{X} X\right) Z_{\mu}^{\prime},
$$


where $g$ 's are gauge couplings, $T^{\alpha}$ and $T^{a}$ are generators of $\mathrm{SU}(3)_{C}$ and $\mathrm{SU}(2)_{L}$, respectively, and $V_{\mu}\left(V=G^{\alpha}, W^{a}, B, Z^{\prime}\right)$ are gauge bosons. The coupling constant $g_{\text {mix }}$ denotes the kinetic mixing between the $\mathrm{U}(1)_{Y}$ and the $\mathrm{U}(1)_{X}$ gauge symmetries, and we will take $g_{\text {mix }}=0$ at the GCU scale. This boundary condition naturally arises from breaking a simple unified gauge group into $\mathrm{SU}(3)_{C} \times \mathrm{SU}(2)_{L} \times \mathrm{U}(1)_{Y} \times \mathrm{U}(1)_{X}$.

We impose the classically scale invariance at the GCU scale, and hence, the scalar potential $V(H, \Phi, S)$ is given by

$$
V(H, \Phi, S)=\lambda_{H}|H|^{4}+\lambda_{\Phi}|\Phi|^{4}+\lambda_{S} S^{4}+\lambda_{H \Phi}|H|^{2}|\Phi|^{2}+\lambda_{H S}|H|^{2} S^{2}+\lambda_{\Phi S}|\Phi|^{2} S^{2},
$$

where there is no dimensionful parameter. In the model, a complex scalar singlet $\Phi$ spontaneously breaks the $\mathrm{U}(1)_{X}$ gauge symmetry due to radiative corrections, i.e. the $\mathrm{CW}$ mechanism. Since the complex scalar field obtains the nonzero vacuum expectation value (VEV), the SM singlet scalar $\Phi$, the $\mathrm{U}(1)_{X}$ gauge boson $Z^{\prime}$, the right-handed neutrinos and the vector-like fermion $N_{L, R}$ become massive. After the $\mathrm{U}(1)_{X}$ symmetry breaking, negative mass terms of a real scalar singlet $S$ and the SM Higgs doublet $H$ are generated, which induces the EW symmetry breaking. Then, $S$, the vector-like fermions and the SM particles become massive, and typically their masses are lighter than those obtained by the $\mathrm{U}(1)_{X}$ symmetry breaking.

Let us explain the symmetry breaking mechanism more explicitly. We consider the CW potential for a classical field of the singlet scalar $\phi$ as

$$
V_{\Phi}(\phi)=\frac{1}{4} \lambda_{\Phi}\left(v_{\Phi}\right) \phi^{4}+\frac{1}{8} \beta_{\lambda_{\Phi}}\left(v_{\Phi}\right) \phi^{4}\left(\ln \frac{\phi^{2}}{v_{\Phi}^{2}}-\frac{25}{6}\right),
$$

where we have taken $\Phi=\phi / \sqrt{2}$ without loss of generality, and $\langle\phi\rangle=v_{\Phi}$ is the VEV of $\phi$. $\beta$ functions of $\Phi, \beta_{\lambda_{\Phi}}$, almost depends on quartic terms of $g_{X}, Y_{M}$ and $Y_{N_{L, R}}$ for $\lambda_{\Phi} \simeq 0$. ( $\beta$ functions of the model parameters are given in appendix.) The effective potential (2.4) satisfies the following renormalization conditions

$$
\left.\frac{\partial^{2} V_{\Phi}}{\partial \phi^{2}}\right|_{\phi=0}=0,\left.\quad \frac{\partial^{4} V_{\Phi}}{\partial \phi^{4}}\right|_{\phi=v_{\Phi}}=6 \lambda_{\Phi}
$$

and the minimization condition of $V_{\Phi}$ induces

$$
\lambda_{\Phi}\left(v_{\Phi}\right) \simeq \frac{11}{6 \pi^{2}}\left[6 g_{X}^{4}\left(v_{\Phi}\right)-\left(\operatorname{tr} Y_{M}^{4}\left(v_{\Phi}\right)+Y_{N_{L}}^{4}\left(v_{\Phi}\right)+Y_{N_{R}}^{4}\left(v_{\Phi}\right)\right)\right]
$$

where we have assumed that the scalar quartic couplings are negligibly small in the righthand side. When this relation is satisfied, the $\mathrm{U}(1)_{X}$ symmetry is broken, and $\Phi$ and $Z^{\prime}$ become massive as

$$
M_{\phi}=\sqrt{\frac{6}{11} \lambda_{\Phi}\left(v_{\Phi}\right)} v_{\Phi}, \quad M_{Z^{\prime}}=2 g_{X}\left(v_{\Phi}\right) v_{\Phi}
$$

respectively. Since the right-hand side of eq. (2.6) should be positive, $\lambda_{\Phi}\left(v_{\Phi}\right) \lesssim g_{X}^{4}\left(v_{\Phi}\right)$ is required, and hence, $M_{\phi}<M_{Z^{\prime}}$ is generally expected. In addition, the quartic terms of Majorana Yukawa couplings $\left(Y_{M}\right.$ and $\left.Y_{N_{L, R}}\right)$ are smaller than the quartic terms of $g_{X}$ 
because of $\lambda_{\Phi}\left(v_{\Phi}\right)>0$. The masses of right-handed neutrinos and $N_{L, R}$ will be discussed in section 3.3 .

After the $\mathrm{U}(1)_{X}$ symmetry breaking, the effective potentials for $s$ and $h$ are approximately given by

$$
V_{S}(s)=\frac{1}{4} \lambda_{S} s^{4}+\frac{1}{4} \lambda_{\Phi} v_{\Phi}^{2} s^{2}, \quad V_{H}(h)=\frac{1}{4} \lambda_{H} h^{4}+\frac{1}{4} \lambda_{H \Phi} v_{\Phi}^{2} h^{2},
$$

where $S=s / \sqrt{2}$ and $H=(0, h / \sqrt{2})^{T}$. Here, we have assumed that $\lambda_{H S}$ are negligibly small compared to $\lambda_{\Phi S}$ and $\lambda_{H \Phi}$ for simplicity. For $\kappa_{1,2} \simeq 0, \lambda_{H S}$ is always negligibly small during renormalization group evolution [see eq. (A.16)]. When $\lambda_{\Phi S}$ and $\lambda_{H \Phi}$ are negative, the nonzero VEVs $\langle s\rangle=v_{S}$ and $\langle h\rangle=v_{H}$ are obtained as

$$
v_{S}^{2}=\frac{-\lambda_{\Phi S}}{2 \lambda_{S}} v_{\Phi}^{2}, \quad v_{H}^{2}=\frac{-\lambda_{H \Phi}}{2 \lambda_{H}} v_{\Phi}^{2}
$$

Note that $v_{S}$ and $v_{H}$ is typically lower than $v_{\Phi}$, because the ratios of quartic couplings $\left(\lambda_{\Phi S} /\left(2 \lambda_{S}\right)\right.$ and $\left.\lambda_{H \Phi} /\left(2 \lambda_{H}\right)\right)$ should be lower than unity to avoid the vacuum instability. The vector-like fermions and the SM particles become massive, while the masses of vectorlike fermions $\left(Q_{L, R}\right.$ and $\left.D_{L, R}\right)$ have to be lower than $1 \mathrm{TeV}$ to realize the GCU as we will show in section 3.1 .

In the end of this section, we mention the $\mathrm{U}(1)_{X}$ breaking scale, which is described by $v_{\Phi}$. Since $M_{Z^{\prime}} / g_{X}>6.9 \mathrm{TeV}$ is required from the LEP-II experiments [49], we obtain the lower bound $v_{\Phi} \gtrsim 3.5 \mathrm{TeV}$. On the other hand, the naturalness of the Higgs mass suggests a relatively small $v_{\Phi}$. A major correction to the Higgs mass is given by $Z^{\prime}$ intermediating diagrams, and one-loop and two-loop corrections are approximately written as

$$
\begin{aligned}
& \Delta m_{h}^{2} \sim \frac{4 x_{H}^{2} g_{X}^{4} v_{\Phi}^{2}}{16 \pi^{2}} \quad \text { for } x_{H} \neq 0, \\
& \Delta m_{h}^{2} \sim \frac{4\left(x_{H}+1\right)\left(4 x_{H}+1\right)}{9} \frac{y_{t}^{2} g_{X}^{4} v_{\Phi}^{2}}{\left(16 \pi^{2}\right)^{2}},
\end{aligned}
$$

respectively. When one defines requirement of the naturalness as $\Delta m_{h}^{2}<M_{h}^{2}$, eqs. (2.10) and (2.11) lead the upper bound on $v_{\Phi}$ as

$$
\begin{aligned}
v_{\Phi} & \lesssim \frac{1}{\left|x_{H}\right|}\left(\frac{0.1}{g_{X}}\right)^{2} \times 10^{5} \mathrm{GeV} \text { for } x_{H} \neq 0 \\
v_{\Phi} & \lesssim\left(\frac{0.1}{g_{X}}\right)^{2} \times 10^{6} \mathrm{GeV}
\end{aligned}
$$

where we have taken $y_{t} \approx 1$. For $\left|x_{H}\right|<0.1$, the two-loop correction gives stronger bound than one-loop correction. In the following, we will use the stronger bound for fixed $x_{H}$. Note that the mass correction from $\Phi$ is always negligible because of a small mixing coupling $\lambda_{H \Phi}$.

\section{Phenomenological and cosmological aspects}

In this section, we will discuss phenomenological and cosmological aspects of the model: the GCU, vacuum stability and triviality, smallness of active neutrino masses, baryon 


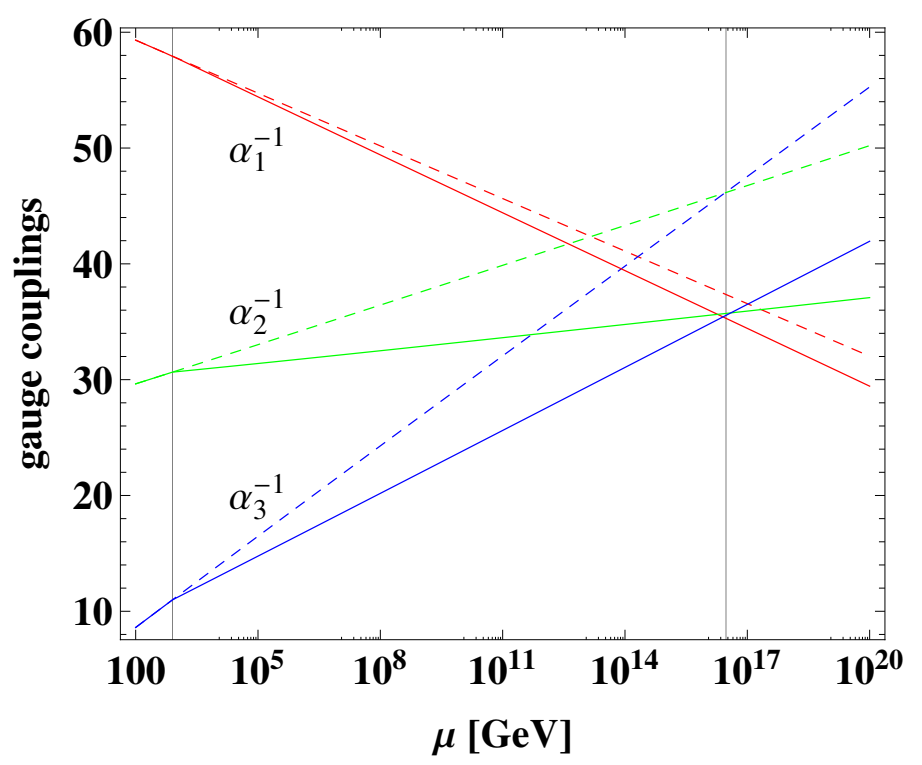

Figure 1. Runnings of gauge couplings $\alpha_{i}^{-1}$. The dashed and solid lines correspond to the SM and the $\mathrm{U}(1)_{X}$ extended model cases, respectively. The vertical lines express $M_{V}=800 \mathrm{GeV}$ and $\Lambda_{\mathrm{GCU}}=3 \times 10^{16} \mathrm{GeV}$.

asymmetry of the universe, and dark matter. We will also restrict the model parameters from the naturalness of the Higgs mass.

\subsection{Gauge coupling unification}

First, we discuss the possibility of the GCU at a high energy scale. Since four additional vector-like fermions $\left(Q_{L, R}\right.$ and $\left.D_{L, R}\right)$ have gauge charges under the SM gauge groups as shown in table 1 , runnings of the SM gauge couplings are modified from the SM. Then, $\beta$ functions of gauge coupling constants are given by

$$
\beta_{g_{Y}}=\frac{g_{Y}^{3}}{16 \pi^{2}} \frac{15}{2}, \quad \beta_{g_{2}}=\frac{g_{2}^{3}}{16 \pi^{2}} \frac{-7}{6}, \quad \beta_{g_{3}}=\frac{g_{3}^{3}}{16 \pi^{2}}(-5),
$$

at 1-loop level. Figure 1 shows runnings of gauge couplings $\alpha_{i}^{-1} \equiv 4 \pi / g_{i}^{2}$, where $\mathrm{U}(1)_{Y}$ gauge coupling is normalized as $g_{1} \equiv \sqrt{5 / 3} g_{Y}$. The calculation has been done for $x_{H}=0$ with using 2-loop RGEs. We note that the running of gauge couplings are almost independent of $x_{H}$. In the figure, the horizontal axis is the renormalization scale and the vertical axis indicates value of $\alpha_{i}^{-1}$. The red, green, and blue lines show $\alpha_{1}^{-1}, \alpha_{2}^{-1}$, and $\alpha_{3}^{-1}$, respectively. The dashed and solid lines correspond to the SM and our model, respectively. The left vertical line stands for a typical scale of vector-like fermions, which has been taken as $M_{V}=800 \mathrm{GeV}$ in figure 1 . For $\mu<M_{V}$, the $\beta$ functions are the SM ones, and we take boundary conditions for the gauge couplings such that experimental values of the Weinberg angle, the fine structure constant, and the strong coupling can be reproduced [50]. The GCU can be achieved at $\Lambda_{\mathrm{GCU}}=(2-4) \times 10^{16} \mathrm{GeV}$, and the unified gauge coupling is $\alpha_{\mathrm{GCU}}^{-1}=(35.4-35.8) .{ }^{3}$ This is the same result as in ref. [34], in which only $Q_{L, R}$ and $D_{L, R}$

\footnotetext{
${ }^{3}$ The GCU can be achieved by adjoint fermions as in ref. [51, 52].
} 


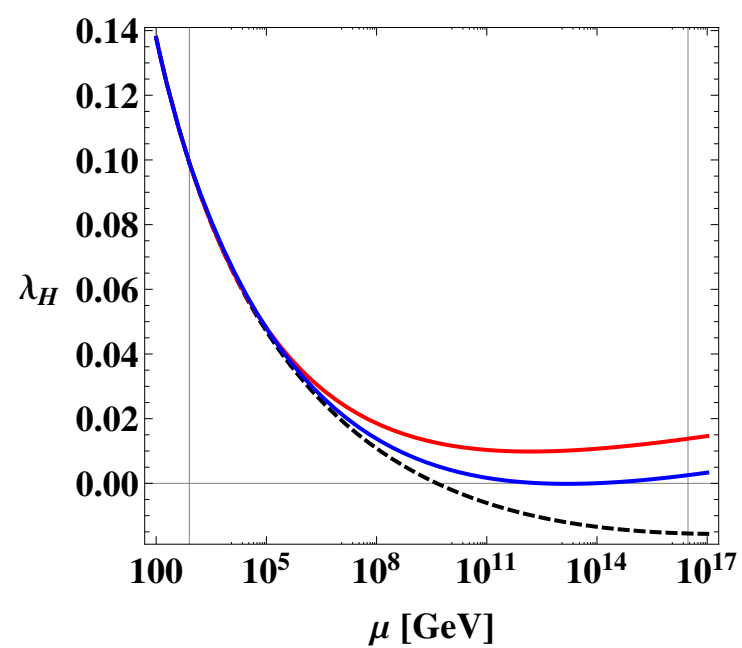

Figure 2. Running of $\lambda_{H}$ in the $\mathrm{U}(1)_{B-L}\left(x_{H}=0\right)$ case. The red and blue lines correspond to $\kappa=0$ and $\kappa=0.33$, respectively. The black dashed line shows running of $\lambda_{H}$ in the SM. The vertical lines express $M_{V}=800 \mathrm{GeV}$ and $\Lambda_{\mathrm{GCU}}=3 \times 10^{16} \mathrm{GeV}$.

are added into the SM. As the vector-like fermion masses become larger, the precision of the GCU becomes worse. Thus, the masses of $Q_{L, R}$ and $D_{L, R}$ should be lighter than $1 \mathrm{TeV}$, while vector-like fermion masses are constrained by the LHC experiments [53-55]. Since the lower bound of vector-like quark lies around $700 \mathrm{GeV}$, the possibility of the GCU can be testable in the near future.

We note that the proton lifetime in a GUT model. The proton lifetime is roughly derived from a four-fermion approximation for the decay channel $p \rightarrow e^{+}+\pi^{0}$, which is given by

$$
\tau_{\mathrm{p}} \sim\left(\alpha_{\mathrm{GCU}}^{-1}\right)^{2} \frac{\Lambda_{\mathrm{GCU}}^{4}}{m_{\mathrm{p}}^{5}}
$$

where $m_{\mathrm{p}}$ is the proton mass. For $\Lambda_{\mathrm{GCU}}=3 \times 10^{16} \mathrm{GeV}$ and $\alpha_{\mathrm{GCU}}^{-1}=35.6$, we can estimate $\tau_{\mathrm{p}} \sim 10^{37} \mathrm{yrs}$, which is much longer than the experimental lower bound $\tau_{\mathrm{p}}>8.2 \times 10^{33}$ yrs [56]. Thus, the model are free from the constraint of the proton decay.

\subsection{Vacuum stability and triviality}

Next, we discuss the vacuum stability. However, it is difficult to investigate exact vacuum stability conditions, since there are three scalar fields and each of them has nonzero VEVs. Therefore, we simply investigate three necessary conditions: $\lambda_{H}>0, \lambda_{\Phi}>0$ and $\lambda_{S}>0$.

The condition $\lambda_{H}>0$ depends on additional contributions to $\beta_{\lambda_{H}}$, i.e., $\kappa_{1,2}, g_{X}$ and scalar mixing couplings. ${ }^{4}$ If their contributions to $\beta_{\lambda_{H}}$ are negligible, since the SM gauge couplings are larger compared to the SM case, running of $\lambda_{H}$ is raised and always positive. For example, however, the EW vacuum becomes instable for $\kappa \gtrsim 0.33$ in the $\mathrm{U}(1)_{B-L}$

\footnotetext{
${ }^{4}$ Running of $\lambda_{H}$ also depends on mass (or Yukawa coupling) of the top quark. We will use the central value of world average, i.e., $M_{t}=173.34 \mathrm{GeV}$ [57]. If we change this value of top quark mass, the following numerical results can slightly change.
} 
$\left(x_{H}=0\right)$ case. We show the running of $\lambda_{H}$ for $x_{H}=0$ in figure 2 , where $\beta_{\lambda_{H}}$ is independent of $g_{X}$ up to the one-loop level, and contributions of $g_{X}$ can be negligible. The red and blue lines correspond to $\kappa=0$ and $\kappa=0.33$, respectively. The black dashed line shows running of $\lambda_{H}$ in the SM. Thus, $\kappa<0.33$ is required to realize the vacuum stability.

The Higgs mass corrections from $Q_{L, R}$ and $D_{L, R}$ loops are given by

$$
\Delta m_{h}^{2} \sim \frac{v_{S}^{2}}{16 \pi^{2}}\left[\left(\kappa_{1}^{2}+\kappa_{2}^{2}\right)\left(f_{Q}^{2}+f_{D}^{2}\right)+2 \kappa_{1} \kappa_{2} f_{Q} f_{D}\right] \sim \frac{12 \kappa^{2} M_{V}^{2}}{16 \pi^{2}},
$$

where we have taken $\kappa=\kappa_{1}=\kappa_{2}$, which naturally arises from $L \leftrightarrow R$ symmetry for the vector-like particles, and $M_{V}=M_{Q}=M_{D}\left(M_{Q}=f_{Q} v_{S} / \sqrt{2}\right.$ and $\left.M_{D}=f_{D} v_{S} / \sqrt{2}\right)$ for simplicity. Then, the naturalness requires $\kappa<0.1$ for $M_{V} \sim 1 \mathrm{TeV}$. Although $\kappa v_{H}$ is a contribution to the vector-like fermion masses from the Higgs, it can be ignored because of $\kappa v_{H} \ll M_{V}$. Since the contribution of $\kappa$ to $\beta_{\lambda_{H}}$, i.e., $24 \lambda_{H} \kappa^{2}-12 \kappa^{4}$, is always positive for $\kappa<0.1$, the naturalness condition also guarantees the vacuum stability. Note that $\kappa \simeq 0$ guarantees $\lambda_{H S} \simeq 0$ at any energy scale, which is required to justify our potential analysis for eq. (2.8).

Here, we check contributions of vector-like fermions to the $S$ and $T$ parameters, which are approximately given by $[58,59]$

$$
\delta S \approx \frac{43}{30 \pi}\left(\frac{\kappa v_{H}}{M_{V}}\right)^{2}, \quad \delta T \approx \frac{3\left(\kappa v_{H}\right)^{2}}{10 \pi \sin ^{2} \theta_{W} M_{W}^{2}}\left(\frac{\kappa v_{H}}{M_{V}}\right)^{2},
$$

where $\theta_{W}$ and $M_{W}$ are the Weinberg angle and the $W$ boson mass, respectively. For $\kappa<0.1$, the parameters are estimated as $\delta S<3 \times 10^{-4}$ and $\delta T<2 \times 10^{-5}$, which are consistent with the precision EW data $S=0.00 \pm 0.08$ and $T=0.05 \pm 0.07$ [56].

The condition $\lambda_{\Phi}>0$ is almost always satisfied when $g_{X}$ is dominant in the right-hand side of eq. (2.6), i.e., $\lambda_{\Phi}\left(v_{\Phi}\right) \sim g_{X}^{4}\left(v_{\Phi}\right)$. In this case, $\beta_{\lambda_{\Phi}}$ is positive up to the GCU scale, and then $\lambda_{\Phi}$ is also positive up to the GCU scale. It is also possible to realize the critical condition $\lambda_{\Phi}\left(\Lambda_{\mathrm{GCU}}\right)=0$ as well as $\lambda_{\Phi}>0$, where the running of $\lambda_{\Phi}$ is curved upward as in the so-called flatland scenario $[9,14,16,21,24]$. Then, both $g_{X}$ and Majorana Yukawa couplings are dominant in $\beta_{\lambda_{\Phi}}$, while $\lambda_{\Phi}$ is much smaller than them. This means that there is a fine-tuning to satisfy eq. (2.6).

When $\lambda_{S}$ is negligible in its $\beta$ function, a solution of its RGE is approximately given by

$$
\lambda_{S}(\mu) \approx \lambda_{S}\left(v_{S}\right)-\frac{1}{16 \pi^{2}}\left(12 f_{Q}^{4}\left(v_{S}\right)+6 f_{D}^{4}\left(v_{S}\right)+2 f_{N}^{4}\left(v_{S}\right)\right) \ln \frac{\mu}{v_{S}},
$$

where $\mu$ is a renormalization scale. Once $v_{S}$ is fixed, $f_{Q}$ and $f_{D}$ are determined to realize the GCU, while $f_{N}$ remains a free parameter. To estimate the condition of $\lambda_{S}>0$, we assume $f_{N}=f_{Q}=f_{D}$ at $\mu=v_{S}$ for simplicity. Then, we can find that $\lambda_{S}$ is positive up to the GCU scale for $\lambda_{S}\left(v_{S}\right) \gtrsim 0.01$. This lower bound of $\lambda_{S}\left(v_{S}\right)$ is almost unchanged for different values of $v_{S}$, because $v_{S}$ dependence is logarithmic.

On the other hand, when $\lambda_{S}$ is dominant in $\beta_{\lambda_{S}}$, the Landau pole might exist, at which the theory is not valid from the point of view of perturbativity (triviality). The energy scale where the Landau pole appears is approximately estimated as

$$
\Lambda_{\mathrm{LP}}=v_{S} \exp \left[\frac{4 \pi^{2} v_{S}^{2}}{9 M_{s}^{2}}\right]
$$




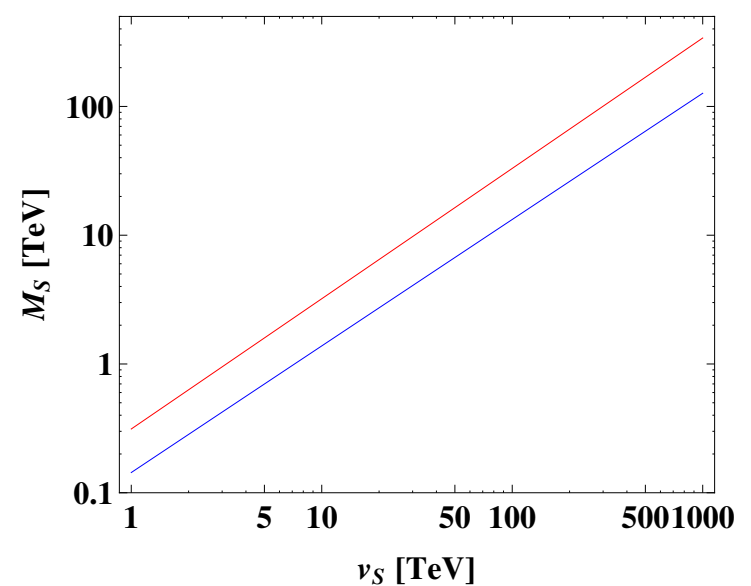

Figure 3. $v_{S}$ dependence on the upper (red) and lower (blue) bounds of $M_{s}$, which correspond to the Landau pole and vacuum stability conditions, respectively. For the Landau pole bound, we take $\Lambda_{\mathrm{LP}}=\Lambda_{\mathrm{GCU}}=3 \times 10^{16} \mathrm{GeV}$ in eq. (3.6).

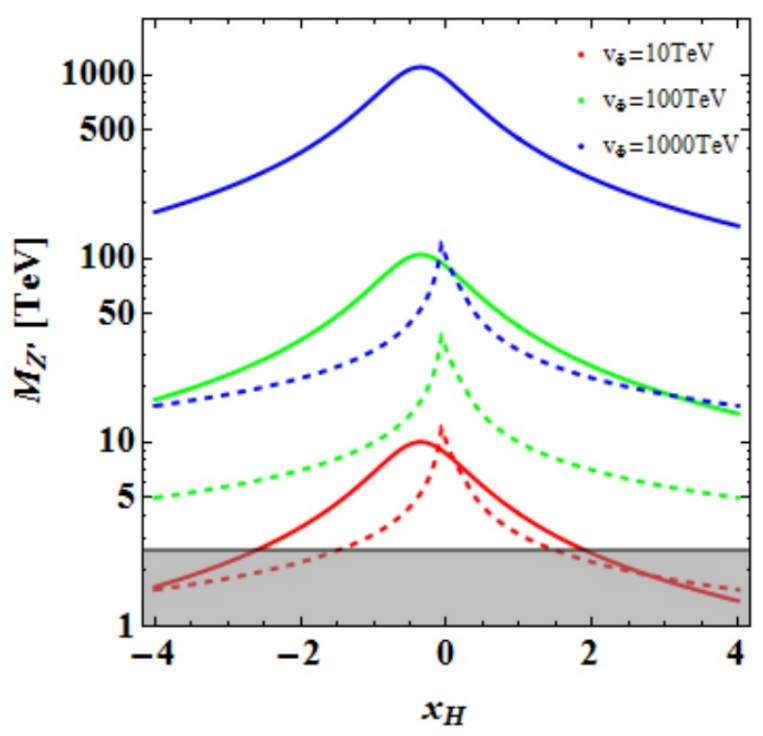

Figure 4. The upper bound of $M_{Z^{\prime}}$ for fixed $v_{\Phi}$, which depends on $x_{H}$. The solid and dashed lines show the Landau pole (3.7) and the naturalness (eqs. (2.12) and (2.13)) bounds, respectively. For the Landau pole bound, we take $\Lambda_{\mathrm{LP}}=\Lambda_{\mathrm{GCU}}=3 \times 10^{16} \mathrm{GeV}$ in eq. (3.7). The shaded region $\left(M_{Z^{\prime}}<2.6 \mathrm{TeV}\right)$ is excluded by the LHC experiments.

where $M_{s}=\sqrt{2 \lambda_{S}\left(v_{S}\right)} v_{S}$ is a mass of the real singlet scalar field. Figure 3 shows $v_{S}$ dependence on the upper (red) and lower (blue) bonds of $M_{s}$, which correspond to the Landau pole and vacuum stability conditions, respectively. Since the both bounds are almost proportional to $v_{S}$, allowed values of $\lambda_{S}\left(v_{S}\right)$ are almost unchanged for different $v_{S}$. We can find a strong constraint for $\lambda_{S}$ as $0.01 \lesssim \lambda_{S}\left(v_{S}\right) \lesssim 0.05$.

In the same way, the Landau pole also exists when $g_{X}\left(v_{\Phi}\right)$ is sufficiently large. The energy scale where the Landau pole appears is approximately estimated by the one-loop 
RGE of $g_{X}$ as

$$
\Lambda_{\mathrm{LP}}=v_{\Phi} \exp \left[\frac{32 \pi^{2} v_{\Phi}^{2}}{\left(44 / 3+64 / 3 x_{H}+30 x_{H}^{2}\right) M_{Z^{\prime}}^{2}}\right]
$$

where $M_{Z^{\prime}}$ is given in eq. (2.7). Figure 4 shows the upper bound of $M_{Z^{\prime}}$ for fixed $v_{\Phi}$, which depends on $x_{H}$. The solid lines show the maximal value of $M_{Z^{\prime}}$ allowed in the model, which are calculated by eq. (3.7) for $\Lambda_{\mathrm{LP}}=\Lambda_{\mathrm{GCU}}=3 \times 10^{16} \mathrm{GeV}$. Note that the peak of solid lines at $x_{H}=-16 / 45$ corresponds to the orthogonal basis of two $\mathrm{U}(1)$ gauges. The dashed lines show the naturalness bound estimated by eqs. (2.12) and (2.13). The red, green, and blue colors correspond to $v_{\Phi}=10,100$, and $1000 \mathrm{TeV}$, respectively. The shaded region $\left(M_{Z^{\prime}}<2.6 \mathrm{TeV}\right)$ is excluded by the LHC experiments [60,61]. When we define the triviality bound as $\Lambda_{\mathrm{GCU}}<\Lambda_{\mathrm{LP}}$, it prohibits the regions above the solid lines. One can see that the bound leads $g_{X}\left(v_{\Phi}\right) \lesssim 0.5$ from eq. (2.7), which is almost independent of $v_{\Phi}$. Since the naturalness requires the stronger constraints than the triviality bound in almost all parameter space, we can say that the naturalness guarantees no Landau pole below the GCU scale. Note that the both bounds are almost the same for $v_{\Phi}=10 \mathrm{TeV}$, and they exclude $M_{Z^{\prime}}>10 \mathrm{TeV}$.

\subsection{Neutrino masses and baryon asymmetry of the universe}

From the Lagrangian (2.1), the neutrino mass terms are given by

$$
\left(\overline{\nu_{L}}, \overline{\nu_{R}^{c}}, \overline{N_{L}}, \overline{N_{R}^{c}}\right)\left(\begin{array}{cccc}
0 & m_{D} & 0 & 0 \\
m_{D}^{T} & M_{M} & 0 & 0 \\
0 & 0 & M_{N_{L}} & m_{N} \\
0 & 0 & m_{N} & M_{N_{R}}
\end{array}\right)\left(\begin{array}{c}
\nu_{L}^{c} \\
\nu_{R} \\
N_{L}^{c} \\
N_{R}
\end{array}\right),
$$

where $m_{D}=Y_{\nu} v_{H} / \sqrt{2}, M_{M}=Y_{M} v_{\Phi} / \sqrt{2}, M_{N_{L, R}}=Y_{N_{L, R}} v_{\Phi} / \sqrt{2}$, and $m_{N}=f_{N} v_{S} / \sqrt{2}$. There is no mixing term between $\nu_{L, R}$ and $N_{L, R}$ due to the $Z_{2}$ symmetry. The active neutrino masses can be obtained by the usual type-I seesaw mechanism [36-40], i.e., $m_{\nu} \approx$ $m_{D} M_{M}^{-1} m_{D}^{T}$. The heavier mass eigenvalue is nearly equal to $M_{M}$, whose upper bound is given by the naturalness of the Higgs mass. Neutrino one-loop diagram contributes the Higgs mass as

$$
\Delta m_{h}^{2} \sim \frac{Y_{\nu}^{2} Y_{M}^{2} v_{\Phi}^{2}}{16 \pi^{2}} \sim \frac{m_{\nu} M_{M}^{3}}{16 \pi^{2} v_{H}^{2}}
$$

where we have used the seesaw relation. For $m_{\nu} \sim 0.1 \mathrm{eV}$, the naturalness requires $M_{M} \lesssim 10^{7} \mathrm{GeV}$

We mention the baryon asymmetry of the universe. In the normal thermal leptogenesis [62], there is a lower bound on the right-handed neutrino mass as $M_{M} \gtrsim 10^{9} \mathrm{GeV}$ [63]. However, the resonant leptogenesis can work even at the $\mathrm{TeV}$ scale, where two right-handed neutrino masses are well-degenerated [41]. In our model, additional $\mathrm{U}(1)_{X}$ gauge interactions make the right-handed neutrinos be in thermal equilibrium with the SM particles [64]. A large efficiency factor can be easily obtained, and the sufficient baryon asymmetry of the 

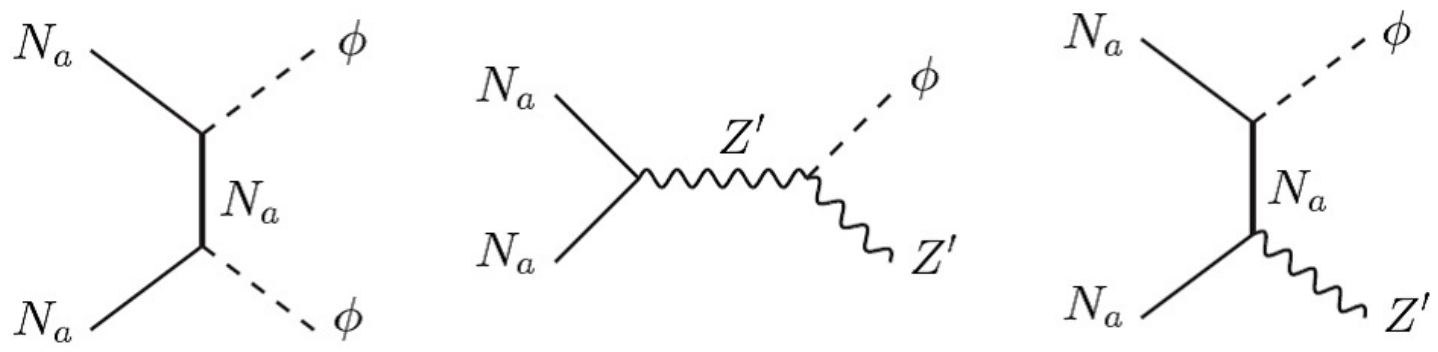

Figure 5. Annihilation processes of the dark matter $N_{a}(a=1,2)$.

universe can be generated by the right-handed neutrinos with a few $\mathrm{TeV}$ masses. Since the neutrino Yukawa coupling $Y_{N}$ and $Y_{M}$ almost do not depend on the other phenomenological problems, we can do the same analysis as in ref. [64], and hence, the result is also the same as in ref. [64].

For the vector-like neutrinos $\left(N_{L, R}\right)$, we consider $M_{N}=M_{N_{L}}=M_{N_{R}}$, which naturally arises from $L \leftrightarrow R$ symmetry for the vector-like fermions. Then, the mass eigenvalues are respectively $M_{N_{1}}=\left|M_{N}-m_{N}\right|$ and $M_{N_{2}}=\left|M_{N}+m_{N}\right|$ for $N_{1}=\left(N_{L}^{c}-N_{R}\right) / \sqrt{2}$ and $N_{2}=\left(N_{L}^{c}+N_{R}\right) / \sqrt{2}$. The lighter mass eigenstate $N_{1}$ is a DM candidate, because its stability is guaranteed by the $Z_{2}$ symmetry. In the limit of $m_{N} \rightarrow 0\left(M_{N_{1}}=M_{N_{2}}\right), N_{1}$ and $N_{2}$ are degenerate, and $N_{2}$ is also effective for a calculation of the DM relic abundance. In the next subsection, we will investigate the degenerate $N_{1,2}$ case.

In our model, the $\mathrm{U}(1)_{X}$ gauge symmetry is successfully achieved via the CW mechanism. It requires $\lambda_{\Phi}\left(v_{\Phi}\right)>0$ in eq. (2.6), that is,

$$
n_{\nu} M_{M}^{4}+2 M_{N}^{4}<\frac{3}{32} M_{Z^{\prime}}^{4}
$$

where $n_{\nu}$ is a relevant number of right-handed neutrinos, which is defined as $\operatorname{tr} Y_{M}^{4}\left(v_{\Phi} / \sqrt{2}\right)^{4}=n_{\nu} M_{M}^{4}$. Thus, the Majorana masses must be lighter than the $Z^{\prime}$ boson mass. We have made sure that this constraint is always satisfied when $N_{1,2}$ explain the DM relic abundance.

\subsection{Dark matter}

To calculate the DM relic abundance, we use the same formula for the DM annihilation cross sections as in ref. [19], where a new vector-like fermion is only $N_{L, R}$ (or $N_{1,2}$ ), and the SM fermions do not have $\mathrm{U}(1)_{X}$ charges. The annihilation processes are $t$-channel $N N \rightarrow \phi \phi, t$ channel $N N \rightarrow Z^{\prime} \phi$, and $Z^{\prime}$ mediated $s$-channel $N N \rightarrow Z^{\prime} \phi$. The corresponding diagrams are shown in figure 5. Although our model has other contributions to the annihilation cross sections, they are all negligible in the following setup. We consider the degenerate case for simplicity, in which there is no vector-like mass term of $N$. Thus, $t$-channel $N N \rightarrow s s$ process and $s$ mediated $s$-channel $N N \rightarrow \nu_{R} \nu_{R}$ process does not occur at tree level. From eq. (3.10), $\left(2 M_{N}\right)^{2}<M_{Z^{\prime}}^{2}$ is always required. Then, the annihilation cross section $\sigma\left(N N \rightarrow Z^{\prime *} \rightarrow f \bar{f}\right)$, where $f$ is some $\mathrm{U}(1)_{X}$ charged fermion, is suppressed by $1 / M_{Z^{\prime}}^{2}$. As a result, we can use the same formula for the DM annihilation cross sections as in ref. [19]. 

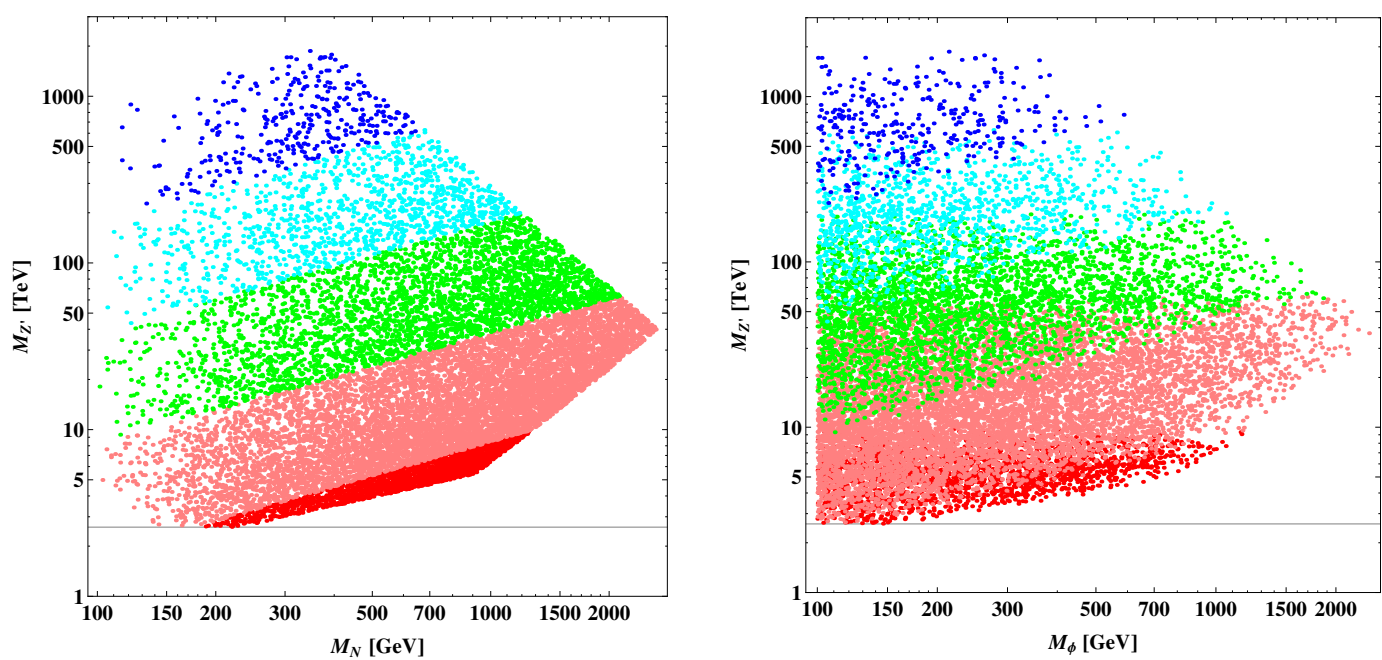

Figure 6. Scatter plots in $\left(M_{N}, M_{Z^{\prime}}\right)$ plane (left) and $\left(M_{\phi}, M_{Z^{\prime}}\right)$ plane (right), which realize the DM relic abundance $\Omega_{\mathrm{DM}} h^{2}=0.1187$, and satisfy all constraints as discussed in this paper as well as the LUX bound. The horizontal line shows the lower bound on $M_{Z^{\prime}}$ by the LHC experiments. The red, pink green, cyan, and blue dots correspond to $6 \mathrm{TeV} \leq v_{\Phi}<10 \mathrm{TeV}, 10 \mathrm{TeV} \leq v_{\Phi}<100 \mathrm{TeV}$, $100 \mathrm{TeV} \leq v_{\Phi}<10^{3} \mathrm{TeV}, 10^{3} \mathrm{TeV} \leq v_{\Phi}<10^{4} \mathrm{TeV}, 10^{4} \mathrm{TeV} \leq v_{\Phi}<10^{5} \mathrm{TeV}$, respectively.

The spin independent cross section for the direct detection is almost dominated by t-channel exchange of scalars $h$ and $\phi$, which has been considered in ref. [19]. However, our model has an additional contribution due to $Z^{\prime}$ exchange diagrams, which is given by [65]

$$
\sigma_{S I}=\frac{m_{n}^{2} M_{N}^{2}}{\pi\left(m_{n}+M_{N}\right)^{2}} \frac{g_{X}^{4}}{M_{Z^{\prime}}^{4}}=7.75 \times 10^{-42}\left(\frac{\mu_{n}}{1 \mathrm{GeV}}\right)^{2}\left(\frac{1 \mathrm{TeV}}{v_{\Phi}}\right)^{4} \mathrm{~cm}^{2},
$$

where $m_{n}$ is the nucleon mass, and $\mu_{n}=m_{n} M_{N} /\left(m_{n}+M_{N}\right)$ is the reduced nucleon mass. For the DM with the masses of $100 \mathrm{GeV}$ and $1 \mathrm{TeV}$, the small $v_{\Phi}$ regions such as $v_{\Phi}<11 \mathrm{TeV}$ and $v_{\Phi}<6 \mathrm{TeV}$ are excluded by the LUX experiment, respectively [67]. These bound are stronger than the LEP bound, where $v_{\Phi}<3.5 \mathrm{TeV}$ is excluded.

In the following, we consider $x_{H}=0\left(\mathrm{U}(1)_{B-L}\right)$ case. There are six new parameters in the model: the $\mathrm{U}(1)_{B-L}$ gauge coupling $g_{X}$, the two Majorana Yukawa coupling $Y_{N_{L}}$, $Y_{N_{R}}$, the two quartic couplings $\lambda_{\Phi}, \lambda_{H \Phi}$, and the VEV of the complex scalar field $v_{\Phi}$. On the other hand, there are two conditions $Y_{N_{L}}=Y_{N_{R}}$ and eq. (2.9), and we require that $N$ explains the DM relic abundance $\Omega_{\mathrm{DM}} h^{2}=0.1187$ [66]. Thus, we have three free parameters for the DM analysis.

Figure 6 shows scatter plots in $\left(M_{N}, M_{Z^{\prime}}\right)$ plane (left) and $\left(M_{\phi}, M_{Z^{\prime}}\right)$ plane (right), which realize the DM relic abundance $\Omega_{\mathrm{DM}} h^{2}=0.1187$, and satisfy all constraints as discussed above as well as the LUX bound. The parameter space starts from the initial values $M_{\phi}=100 \mathrm{GeV}, M_{N}=100 \mathrm{GeV}$, and $M_{Z^{\prime}}=2.6 \mathrm{TeV}$. Although the two figures in figure 6 are very similar, $M_{N}>M_{\phi}$ is always satisfied. The region of $M_{Z^{\prime}}<2.6 \mathrm{TeV}$ is excluded by the current LHC bound [60,61]. Since $g_{X} \lesssim 0.5$ is required to avoid the Landau pole, the upper bound on $M_{Z^{\prime}}$ is given by $M_{Z^{\prime}} \lesssim v_{\Phi}$, while the upper bound in the $M_{N, \phi} \gtrsim 500 \mathrm{GeV}$ region is given by the naturalness $(2.13)$. In the $200 \mathrm{GeV} \lesssim M_{N, \phi} \lesssim$ 
$900 \mathrm{GeV}$ region, the lower bound on $M_{Z^{\prime}}$ is given by the LUX bound. To realize the DM relic abundance, sufficiently large annihilation cross sections are required, which induce the lower bound on $M_{Z^{\prime}}$ in the $M_{N} \gtrsim 900 \mathrm{GeV}$ region. From figure 6 , we can see the upper bound on the DM mass as $M_{N} \lesssim 2.6 \mathrm{TeV}$, and the bound of $M_{\phi}$ is almost the same as $M_{N}$.

\section{Conclusion}

To solve the gauge hierarchy problem, we have constructed a classically scale invariant model with a $\mathrm{U}(1)_{X}$ gauge extension. We have assumed the classical scale invariance at the GCU scale, where the Higgs mass completely vanishes even with some quantum corrections. The scale invariance is violated around the $\mathrm{TeV}$ scale by the $\mathrm{CW}$ mechanism, and the Higgs mass can be naturally generated through the scalar mixing term. The GCU is realized by vector-like fermions $Q_{L, R}$ and $D_{L, R}$, which respectively have the same quantum number as the SM quark doublet and down-type quark singlet but distinguished by the additional $Z_{2}$ symmetry, and their masses lie in $800 \mathrm{GeV} \lesssim M_{V} \lesssim 1 \mathrm{TeV}$. The GCU scale is $\Lambda_{\mathrm{GCU}}=3 \times 10^{16} \mathrm{GeV}$ with $\alpha_{\mathrm{GCU}}^{-1}=35.6$, and the proton life time is estimated as $\tau_{\mathrm{p}} \sim 10^{37} \mathrm{yrs}$, which is much longer than the experimental lower bound $\tau_{\mathrm{p}}>8.2 \times 10^{33} \mathrm{yrs}$.

In addition, we have shown that the model can explain the vacuum stability, smallness of active neutrino masses, baryon asymmetry of the universe, and dark matter relic abundance without inducing large Higgs mass corrections. Since there are additional fermions with the SM gauge charges, the SM gauge couplings become larger than the SM case, which leads smaller top Yukawa couplings. Then, the $\beta$ function of the Higgs quartic coupling becomes larger, and hence the EW vacuum becomes stable. The smallness of active neutrino masses and the baryon asymmetry of the universe can be explained by the righthanded neutrinos via the type-I seesaw mechanism and resonant leptogenesis, respectively. The DM candidate is the SM singlet Majorana fermions $N_{1,2}$, and stability of the DM is guaranteed by the additional $Z_{2}$ symmetry. We have analyzed the DM relic abundance in the degenerate case $\left(M_{N_{1}}=M_{N_{2}}\right)$, and found the upper bound on the DM mass as $M_{N} \lesssim 2.6 \mathrm{TeV}$.

\section{Acknowledgments}

The authors thank Y. Kawamura for helpful discussion on the origin of the vector-like fermions. This work is partially supported by Scientific Grants by the Ministry of Education, Culture, Sports, Science and Technology (Nos. 24540272, 26247038, and 15H01037). The work of Y.Y. is supported by Research Fellowships of the Japan Society for the Promotion of Science for Young Scientists (Grants No. 26·2428).

\section{A $\beta$ functions in the $\mathrm{U}(1)_{X}$ extended $\mathrm{SM}$}

We give one-loop $\beta$-functions in our model:

$$
\begin{aligned}
& \beta_{g_{Y}}=\frac{g_{Y}^{3}}{16 \pi^{2}} \frac{15}{2}, \quad \beta_{g_{2}}=\frac{g_{2}^{3}}{16 \pi^{2}} \frac{-7}{6}, \quad \beta_{g_{3}}=\frac{g_{3}^{3}}{16 \pi^{2}}(-5), \\
& \beta_{g_{X}}=\frac{g_{X}}{16 \pi^{2}}\left[\left(\frac{44}{3}+\frac{64}{3} x_{H}+30 x_{H}^{2}\right) g_{X}^{2}+\frac{15}{2} g_{\text {mix }}^{2}+\left(\frac{32}{3}+30 x_{H}\right) g_{\text {mix }} g_{X}\right],
\end{aligned}
$$




$$
\begin{aligned}
& \beta_{g_{\text {mix }}}=\frac{1}{16 \pi^{2}}\left[g_{\text {mix }}\left(\frac{15}{2}\left(g_{\text {mix }}^{2}+2 g_{Y}^{2}\right)+\left(\frac{44}{3}+\frac{64}{3} x_{H}+30 x_{H}^{2}\right) g_{X}^{2}\right)\right. \\
& \left.+\left(\frac{32}{3}+30 x_{H}\right) g_{X}\left(g_{Y}^{2}+g_{\text {mix }}^{2}\right)\right] \\
& \beta_{y_{t}}=\frac{y_{t}}{16 \pi^{2}}\left[\frac{9}{2} y_{t}^{2}-\left(8 g_{3}^{2}+\frac{9}{4} g_{2}^{2}+\frac{17}{12}\left(g_{Y}^{2}+g_{\text {mix }}^{2}\right)+\left(\frac{2}{3}+\frac{10}{3} x_{H}+\frac{17}{3} x_{H}^{2}\right) g_{X}^{2}\right.\right. \\
& \left.\left.+\left(\frac{5}{3}+\frac{17}{3} x_{H}\right) g_{\text {mix }} g_{X}\right)+3\left(\kappa_{1}^{2}+\kappa_{2}^{2}\right)\right], \\
& \beta_{Y_{M}}=\frac{Y_{M}}{16 \pi^{2}}\left[4 Y_{M}^{2}+2 \operatorname{tr} Y_{M}^{2}+2\left(Y_{N_{L}}^{2}+Y_{N_{R}}^{2}\right)-6 g_{X}^{2}\right], \\
& \beta_{Y_{N_{L}}}=\frac{1}{16 \pi^{2}}\left[Y_{N_{L}}\left(6 Y_{N_{L}}^{2}+f_{N}^{2}+2\left(\operatorname{tr} Y_{M}^{2}+Y_{N_{R}}^{2}\right)-6 g_{X}^{2}\right)+2 f_{N}^{2} Y_{N_{R}}\right] \text {, } \\
& \beta_{Y_{N_{R}}}=\frac{1}{16 \pi^{2}}\left[Y_{N_{R}}\left(6 Y_{N_{R}}^{2}+f_{N}^{2}+2\left(\operatorname{tr} Y_{M}^{2}+Y_{N_{L}}^{2}\right)-6 g_{X}^{2}\right)+2 f_{N}^{2} Y_{N_{L}}\right] \text {, } \\
& \beta_{\kappa_{1}}=\frac{1}{16 \pi^{2}}\left[\kappa _ { 1 } \left(-8 g_{3}^{2}-\frac{9}{4} g_{2}^{2}-\frac{5}{12}\left(g_{Y}^{2}+g_{\text {mix }}^{2}\right)+\frac{1}{3}\left(1-5 x_{H}\right) g_{\text {mix }} g_{X}\right.\right. \\
& \left.\left.\left.+\frac{1}{3}\left(-2+2 x_{H}-5 x_{H}^{2}\right) g_{X}^{2}+\frac{1}{2}\left(f_{Q}^{2}+f_{D}^{2}\right)+3 y_{t}^{2}+\frac{9}{2} \kappa_{1}^{2}+3 \kappa_{2}^{2}\right)+2 f_{Q} f_{D} \kappa_{(2)}\right] .8\right) \\
& \beta_{\kappa_{2}}=\frac{1}{16 \pi^{2}}\left[\kappa _ { 2 } \left(-8 g_{3}^{2}-\frac{9}{4} g_{2}^{2}-\frac{5}{12}\left(g_{Y}^{2}+g_{\text {mix }}^{2}\right)+\frac{1}{3}\left(1-5 x_{H}\right) g_{\text {mix }} g_{X}\right.\right. \\
& \left.\left.+\frac{1}{3}\left(-2+2 x_{H}-5 x_{H}^{2}\right) g_{X}^{2}+\frac{1}{2}\left(f_{Q}^{2}+f_{D}^{2}\right)+3 y_{t}^{2}+\frac{9}{2} \kappa_{2}^{2}+3 \kappa_{1}^{2}\right)+2 f_{Q} f_{D} \kappa_{1}\right], \\
& \beta_{f_{Q}}=\frac{1}{16 \pi^{2}}\left[f _ { Q } \left(-8 g_{3}^{2}-\frac{9}{2} g_{2}^{2}-\frac{1}{6}\left(g_{Y}^{2}+g_{\text {mix }}^{2}\right)-\frac{2}{3}\left(1+x_{H}\right) g_{\text {mix }} g_{X}\right.\right. \\
& \left.\left.-\frac{2}{3}\left(1+x_{H}\right)^{2} g_{X}^{2}+\frac{1}{2}\left(\kappa_{1}^{2}+\kappa_{2}^{2}\right)+15 f_{Q}^{2}+6 f_{D}^{2}+2 f_{N}^{2}\right)+2 f_{D} \kappa_{1} \kappa_{2}\right], \\
& \beta_{f_{D}}=\frac{1}{16 \pi^{2}}\left[f _ { D } \left(-8 g_{3}^{2}-\frac{2}{3}\left(g_{Y}^{2}+g_{\text {mix }}^{2}\right)+\frac{4}{3}\left(1-2 x_{H}\right) g_{\text {mix }} g_{X}-\frac{2}{3}\left(1-2 x_{H}\right)^{2} g_{X}^{2}\right.\right. \\
& \left.\left.+\left(\kappa_{1}^{2}+\kappa_{2}^{2}\right)+12 f_{Q}^{2}+9 f_{D}^{2}+2 f_{N}^{2}\right)+4 f_{Q} \kappa_{1} \kappa_{2}\right], \\
& \beta_{f_{N}}=\frac{f_{N}}{16 \pi^{2}}\left[-6 g_{X}^{2}+2 Y_{N_{L}}^{2}+8 Y_{N_{L}} Y_{N_{R}}+2 Y_{N_{R}}^{2}+12 f_{Q}^{2}+6 f_{D}^{2}+5 f_{N}^{2}\right], \\
& \beta_{\lambda_{H}}=\frac{1}{16 \pi^{2}}\left[24 \lambda_{H}^{2}+\lambda_{H \Phi}^{2}+2 \lambda_{H S}^{2}+\lambda_{H}\left(12 y_{t}^{2}-9 g_{2}^{2}-3\left(g_{Y}^{2}+g_{\text {mix }}^{2}\right)-12 x_{H}^{2} g_{X}^{2}\right.\right. \\
& \left.-12 x_{H} g_{\text {mix }} g_{X}+12\left(\kappa_{1}^{2}+\kappa_{2}^{2}\right)\right)-6 y_{t}^{4}-6\left(\kappa_{1}^{4}+\kappa_{2}^{4}\right)+\frac{3}{8}\left\{2 g_{2}^{4}+\left(g_{2}^{2}+\left(g_{Y}^{2}+g_{\text {mix }}^{2}\right)\right)^{2}\right\} \\
& \left.+3 x_{H} g_{\text {mix }} g_{X}\left(g_{2}^{2}+g_{Y}^{2}+g_{\text {mix }}^{2}+4 x_{H}^{2} g_{X}^{2}\right)+3 x_{H}^{2} g_{X}^{2}\left(g_{2}^{2}+g_{Y}^{2}+3 g_{\text {mix }}^{2}+2 x_{H}^{2} g_{X}^{2}\right)\right] \text {, }
\end{aligned}
$$$$
\beta_{\lambda_{\Phi}}=\frac{1}{16 \pi^{2}}\left[20 \lambda_{\Phi}^{2}+2 \lambda_{H \Phi}^{2}+2 \lambda_{\Phi S}^{2}+\lambda_{\Phi}\left(8\left(\operatorname{tr} Y_{M}^{2}+Y_{N_{L}}^{2}+Y_{N_{R}}^{2}\right)-48 g_{X}^{2}\right)+96 g_{X}^{4}\right.
$$$$
\left.-16\left(\operatorname{tr} Y_{M}^{4}+Y_{N_{L}}^{4}+Y_{N_{R}}^{4}\right)\right]
$$$$
\beta_{\lambda_{S}}=\frac{1}{16 \pi^{2}}\left[72 \lambda_{S}^{2}+2 \lambda_{H S}^{2}+\lambda_{\Phi S}^{2}+\lambda_{S}\left(48 f_{Q}^{2}+24 f_{D}^{2}+8 f_{N}^{2}\right)-12 f_{Q}^{4}-6 f_{D}^{4}-2 f_{N}^{4}\right],
$$ 


$$
\begin{aligned}
\beta_{\lambda_{H \Phi}}=\frac{1}{16 \pi^{2}}[ & 4 \lambda_{H S} \lambda_{\Phi S}+\lambda_{H \Phi}\left(12 \lambda_{H}+8 \lambda_{\Phi}+4 \lambda_{H \Phi}-\frac{9}{2} g_{2}^{2}-\frac{3}{2}\left(g_{Y}^{2}+g_{\text {mix }}^{2}\right)\right. \\
& \left.-6\left(4+x_{H}^{2}\right) g_{X}^{2}-6 x_{H} g_{\text {mix }} g_{X}+6 y_{t}^{2}+6\left(\kappa_{1}^{2}+\kappa_{2}^{2}\right)+4\left(\operatorname{tr} Y_{M}^{2}+Y_{N_{L}}^{2}+Y_{N_{R}}^{2}\right)\right) \\
& \left.+12 g_{X}^{2}\left(g_{\text {mix }}+2 x_{H} g_{X}\right)^{2}\right]
\end{aligned}
$$

Open Access. This article is distributed under the terms of the Creative Commons Attribution License (CC-BY 4.0), which permits any use, distribution and reproduction in any medium, provided the original author(s) and source are credited.

\section{References}

[1] ATLAS, CMS collaboration, Combined measurement of the Higgs boson mass in pp collisions at $\sqrt{s}=7$ and $8 \mathrm{TeV}$ with the ATLAS and CMS experiments, Phys. Rev. Lett. 114 (2015) 191803 [arXiv: 1503.07589] [INSPIRE].

[2] R. Hempfling, The next-to-minimal Coleman-Weinberg model, Phys. Lett. B 379 (1996) 153 [hep-ph/9604278] [INSPIRE].

[3] W.-F. Chang, J.N. Ng and J.M.S. Wu, Shadow Higgs from a scale-invariant hidden U(1)(s) model, Phys. Rev. D 75 (2007) 115016 [hep-ph/0701254] [INSPIRE].

[4] S. Iso, N. Okada and Y. Orikasa, The minimal B-L model naturally realized at TeV scale, Phys. Rev. D 80 (2009) 115007 [arXiv:0909.0128] [InSPIRE].

[5] S. Iso, N. Okada and Y. Orikasa, Classically conformal B-L extended standard model, Phys. Lett. B 676 (2009) 81 [arXiv:0902 .4050] [INSPIRE].

[6] N. Okada and Y. Orikasa, Dark matter in the classically conformal B-L model, Phys. Rev. D 85 (2012) 115006 [arXiv: 1202.1405] [INSPIRE].

[7] S. Iso and Y. Orikasa, TeV scale B-L model with a flat Higgs potential at the Planck scale In view of the hierarchy problem, PTEP 2013 (2013) 023B08 [arXiv:1210.2848] [INSPIRE].

[8] C. Englert, J. Jaeckel, V.V. Khoze and M. Spannowsky, Emergence of the electroweak scale through the Higgs portal, JHEP 04 (2013) 060 [arXiv:1301.4224] [INSPIRE].

[9] E.J. Chun, S. Jung and H.M. Lee, Radiative generation of the Higgs potential, Phys. Lett. B $\mathbf{7 2 5}$ (2013) 158 [Erratum ibid. B $\mathbf{7 3 0}$ (2014) 357] [arXiv:1304.5815] [INSPIRE].

[10] M. Heikinheimo, A. Racioppi, M. Raidal, C. Spethmann and K. Tuominen, Physical naturalness and dynamical breaking of classical scale invariance, Mod. Phys. Lett. A 29 (2014) 1450077 [arXiv:1304.7006] [INSPIRE]. 
[11] I. Oda, Classically scale-invariant B-L model and conformal gravity, Phys. Lett. B 724 (2013) 160 [arXiv: 1305.0884] [INSPIRE].

[12] E. Gabrielli, M. Heikinheimo, K. Kannike, A. Racioppi, M. Raidal and C. Spethmann, Towards completing the standard model: vacuum stability, EWSB and dark matter, Phys. Rev. D 89 (2014) 015017 [arXiv: 1309.6632] [INSPIRE].

[13] V.V. Khoze and G. Ro, Leptogenesis and neutrino oscillations in the classically conformal standard model with the Higgs portal, JHEP 10 (2013) 075 [arXiv:1307.3764] [INSPIRE].

[14] M. Hashimoto, S. Iso and Y. Orikasa, Radiative symmetry breaking at the Fermi scale and flat potential at the Planck scale, Phys. Rev. D 89 (2014) 016019 [arXiv:1310.4304] [INSPIRE].

[15] M. Lindner, D. Schmidt and A. Watanabe, Dark matter and $\mathrm{U}(1)^{\prime}$ symmetry for the right-handed neutrinos, Phys. Rev. D 89 (2014) 013007 [arXiv:1310.6582] [INSPIRE].

[16] M. Hashimoto, S. Iso and Y. Orikasa, Radiative symmetry breaking from flat potential in various U(1)' models, Phys. Rev. D 89 (2014) 056010 [arXiv:1401.5944] [INSPIRE].

[17] S. Benic and B. Radovcic, Electroweak breaking and dark matter from the common scale, Phys. Lett. B 732 (2014) 91 [arXiv:1401.8183] [INSPIRE].

[18] V.V. Khoze, C. McCabe and G. Ro, Higgs vacuum stability from the dark matter portal, JHEP 08 (2014) 026 [arXiv: 1403.4953] [INSPIRE].

[19] S. Benic and B. Radovcic, Majorana dark matter in a classically scale invariant model, JHEP 01 (2015) 143 [arXiv: 1409.5776] [INSPIRE].

[20] H. Okada and Y. Orikasa, Classically conformal radiative neutrino model with gauged $B-L$ symmetry, arXiv:1412.3616 [INSPIRE].

[21] K. Kawana, Criticality and inflation of the gauged B-L model, PTEP 2015 (2015) $073 \mathrm{~B} 04$ [arXiv: 1501.04482] [INSPIRE].

[22] J. Guo, Z. Kang, P. Ko and Y. Orikasa, Accidental dark matter: case in the scale invariant local B-L model, Phys. Rev. D 91 (2015) 115017 [arXiv:1502.00508] [INSPIRE].

[23] P. Humbert, M. Lindner and J. Smirnov, The inverse seesaw in conformal electro-weak symmetry breaking and phenomenological consequences, JHEP 06 (2015) 035 [arXiv: 1503.03066] [INSPIRE].

[24] N. Haba and Y. Yamaguchi, Vacuum stability in the $U(1)_{\chi}$ extended model with vanishing scalar potential at the Planck scale, PTEP 2015 (2015) 093B05 [arXiv: 1504.05669] [INSPIRE].

[25] S. Oda, N. Okada and D.S. Takahashi, Classically conformal U(1)' extended standard model and Higgs vacuum stability, Phys. Rev. D 92 (2015) 015026 [arXiv: 1504.06291] [INSPIRE].

[26] P. Humbert, M. Lindner, S. Patra and J. Smirnov, Lepton number violation within the conformal inverse seesaw, JHEP 09 (2015) 064 [arXiv: 1505.07453] [INSPIRE].

[27] A.D. Plascencia, Classical scale invariance in the inert doublet model, JHEP 09 (2015) 026 [arXiv: 1507.04996] [INSPIRE].

[28] A. Karam and K. Tamvakis, Dark matter and neutrino masses from a scale-invariant multi-Higgs portal, Phys. Rev. D 92 (2015) 075010 [arXiv:1508.03031] [InSPIRE].

[29] A. Das, N. Okada and N. Papapietro, Electroweak vacuum stability in classically conformal $B-L$ extension of the standard model, arXiv:1509.01466 [INSPIRE].

[30] S.R. Coleman and E.J. Weinberg, Radiative corrections as the origin of spontaneous symmetry breaking, Phys. Rev. D 7 (1973) 1888 [INSPIRE].

[31] W.A. Bardeen, On naturalness in the standard model, FERMILAB-CONF-95-391 (1995). 
[32] G.F. Giudice and A. Romanino, Split supersymmetry, Nucl. Phys. B 699 (2004) 65 [Erratum ibid. B 706 (2005) 65] [hep-ph/0406088] [INSPIRE].

[33] N. Haba, H. Ishida, R. Takahashi and Y. Yamaguchi, Hierarchy problem, gauge coupling unification at the Planck scale and vacuum stability, Nucl. Phys. B 900 (2015) 244 [arXiv: 1412.8230] [INSPIRE].

[34] I. Gogoladze, B. He and Q. Shafi, New fermions at the LHC and mass of the Higgs boson, Phys. Lett. B 690 (2010) 495 [arXiv: 1004.4217] [INSPIRE].

[35] F. Bazzocchi and M. Fabbrichesi, Little hierarchy problem for new physics just beyond the LHC, Phys. Rev. D 87 (2013) 036001 [arXiv:1212.5065] [InSPIRE].

[36] P. Minkowski, $\mu \rightarrow$ er at a rate of one out of $10^{9}$ muon decays?, Phys. Lett. B 67 (1977) 421 [INSPIRE].

[37] T. Yanagida, Horizontal symmetry and masses of neutrinos, in the proceedings of the Workshop on unified theory and baryon number in the universe, O. Sawada and A. Sugamoto eds., KEK, Tsukuba, Japan (1979).

[38] M. Gell-Mann, P. Ramond, R. Slansky, Complex spinors and unified theories, in Supergravity, D.Z. Freedman and P.van Nieuwenhuizen eds., North Holland, Amsterdam, The Netherlands (1979).

[39] S.L. Glashow, The future of elementary particle physics, in Quarks and leptons, Cargèse lectures, M. Lévy et al. eds., Plenum Press, New York, U.S.A. (1980).

[40] R.N. Mohapatra and G. Senjanović, Neutrino mass and spontaneous parity violation, Phys. Rev. Lett. 44 (1980) 912 [INSPIRE].

[41] A. Pilaftsis and T.E.J. Underwood, Resonant leptogenesis, Nucl. Phys. B 692 (2004) 303 [hep-ph/0309342] [INSPIRE].

[42] N. Okada and O. Seto, Higgs portal dark matter in the minimal gauged $U(1)_{B-L}$ model, Phys. Rev. D 82 (2010) 023507 [arXiv: 1002.2525] [inSPIRE].

[43] P.H. Frampton and C. Vafa, Conformal approach to particle phenomenology, hep-th/9903226 [INSPIRE].

[44] P.H. Frampton, Conformality and gauge coupling unification, Phys. Rev. D 60 (1999) 085004 [hep-th/9905042] [INSPIRE].

[45] P.H. Frampton, R.N. Mohapatra and S. Suh, Prediction of $\sin ^{2} \theta(W)$ in a conformal approach to coupling unification, Phys. Lett. B 520 (2001) 331 [hep-ph/0104211] [INSPIRE].

[46] M. Shaposhnikov and C. Wetterich, Asymptotic safety of gravity and the Higgs boson mass, Phys. Lett. B 683 (2010) 196 [arXiv:0912.0208] [InSPIRE].

[47] N. Haba, K. Kaneta, R. Takahashi and Y. Yamaguchi, Gravitational effects on vanishing Higgs potential at the Planck scale, Phys. Rev. D 91 (2015) 016004 [arXiv:1408.5548] [INSPIRE].

[48] G. Barenboim, F.J. Botella and O. Vives, Constraining models with vector-like fermions from FCNC in $K$ and B physics, Nucl. Phys. B 613 (2001) 285 [hep-ph/0105306] [INSPIRE].

[49] M. Carena, A. Daleo, B.A. Dobrescu and T.M.P. Tait, $Z^{\prime}$ gauge bosons at the Tevatron, Phys. Rev. D 70 (2004) 093009 [hep-ph/0408098] [INSPIRE].

[50] D. Buttazzo et al., Investigating the near-criticality of the Higgs boson, JHEP 12 (2013) 089 [arXiv: 1307.3536] [INSPIRE].

[51] N. Haba, K. Kaneta and R. Takahashi, Next to new minimal standard model, Phys. Lett. B 734 (2014) 220 [arXiv: 1309.1231] [INSPIRE]. 
[52] N. Haba, K. Kaneta and R. Takahashi, NNMSM type-II and -III, Eur. Phys. J. C 74 (2014) 2696 [arXiv: 1309. 3254] [INSPIRE].

[53] CMS collaboration, Search for heavy lepton partners of neutrinos in proton-proton collisions in the context of the type-III seesaw mechanism, Phys. Lett. B 718 (2012) 348 [arXiv: 1210.1797] [INSPIRE].

[54] CMS collaboration, Inclusive search for a vector-like T quark with charge $\frac{2}{3}$ in pp collisions at $\sqrt{s}=8 \mathrm{TeV}$, Phys. Lett. B $\mathbf{7 2 9}$ (2014) 149 [arXiv:1311.7667] [INSPIRE].

[55] ATLAS collaboration, Search for pair and single production of new heavy quarks that decay to a $Z$ boson and a third-generation quark in pp collisions at $\sqrt{s}=8$ TeV with the ATLAS detector, JHEP 11 (2014) 104 [arXiv:1409.5500] [INSPIRE].

[56] Particle Data Group collaboration, K.A. Olive et al., Review of particle physics, Chin. Phys. C 38 (2014) 090001 [inSPIRE].

[57] ATLAS, CDF, CMS, D0 collaboration, First combination of Tevatron and LHC measurements of the top-quark mass, arXiv:1403.4427 [INSPIRE].

[58] L. Lavoura and J.P. Silva, The oblique corrections from vector-like singlet and doublet quarks, Phys. Rev. D 47 (1993) 2046 [INSPIRE].

[59] N. Maekawa, Electroweak symmetry breaking by vector-like fermions' condensation with small $S$ and T parameters, Phys. Rev. D 52 (1995) 1684 [INSPIRE].

[60] ATLAS collaboration, Search for high-mass dilepton resonances in pp collisions at $\sqrt{s}=8 \mathrm{TeV}$ with the ATLAS detector, Phys. Rev. D 90 (2014) 052005 [arXiv:1405.4123] [INSPIRE].

[61] CMS collaboration, Search for physics beyond the standard model in dilepton mass spectra in proton-proton collisions at $\sqrt{s}=8 \mathrm{TeV}$, JHEP 04 (2015) 025 [arXiv:1412.6302] [INSPIRE].

[62] M. Fukugita and T. Yanagida, Baryogenesis without grand unification, Phys. Lett. B 174 (1986) 45 [INSPIRE].

[63] S. Davidson and A. Ibarra, A lower bound on the right-handed neutrino mass from leptogenesis, Phys. Lett. B 535 (2002) 25 [hep-ph/0202239] [INSPIRE].

[64] S. Iso, N. Okada and Y. Orikasa, Resonant leptogenesis in the minimal B-L extended standard model at TeV, Phys. Rev. D 83 (2011) 093011 [arXiv: 1011.4769] [InSPIRE].

[65] M. Duerr, P. Fileviez Perez and J. Smirnov, Simplified Dirac dark matter models and gamma-ray lines, Phys. Rev. D 92 (2015) 083521 [arXiv: 1506.05107] [INSPIRE].

[66] Planck collaboration, P.A.R. Ade et al., Planck 2013 results. XVI. Cosmological parameters, Astron. Astrophys. 571 (2014) A16 [arXiv:1303.5076] [INSPIRE].

[67] LUX collaboration, D.S. Akerib et al., First results from the LUX dark matter experiment at the Sanford Underground Research Facility, Phys. Rev. Lett. 112 (2014) 091303 [arXiv: 1310.8214] [INSPIRE]. 